\title{
Biocompatible hydrogel membranes for the protection of RNA aptamer- based electrochemical sensors
}

Lauren R. Schoukroun-Barnes, Samiullah Wagan, Juan Liu, Jennie B. Leach, Ryan J. White

Lauren R. Schoukroun-Barnes, Samiullah Wagan, Juan Liu, Jennie B. Leach, Ryan J. White, "Biocompatible hydrogel membranes for the protection of RNA aptamer-based electrochemical sensors ," Proc. SPIE 8719, Smart Biomedical and Physiological Sensor Technology X, 871901 (31 May 2013); doi: $10.1117 / 12.2016020$

Event: SPIE Defense, Security, and Sensing, 2013, Baltimore, Maryland, United States 


\title{
Biocompatible Hydrogel Membranes for the Protection of RNA Aptamer- Based Electrochemical Sensors
}

\author{
Lauren R. Schoukroun-Barnes, ${ }^{1}$ Samiullah Wagan, ${ }^{1}$ Juan Liu, ${ }^{1}$ Jennie B. Leach, ${ }^{2}$ Ryan J. White, ${ }^{1}$ \\ ${ }^{1}$ Electrochemistry Laboratory, Department of Chemistry and Biochemistry, University of Maryland \\ Baltimore County (UMBC) \\ ${ }^{2}$ Chemical, Biochemical \& Environmental Engineering, University of Maryland Baltimore County \\ (UMBC)
}

\begin{abstract}
Electrochemical-aptamer based (E-AB) sensors represent a universal specific, selective, and sensitive sensing platform for the detection of small molecule targets. Their specific detection abilities are afforded by oligonucleotide (RNA or DNA) aptamers employed as electrode-bound biorecognition elements. Sensor signaling is predicated on bindinginduced changes in conformation and/or flexibility of the aptamer that is readily measurable electrochemically. While sensors fabricated using DNA aptamers can achieve specific and selective detection even in unadulterated sample matrices, such as blood serum, RNA-based sensors fail when challenged in the same sample matrix without significant sample pretreatment. This failure is at least partially a result of enzymatic degradation of the RNA sensing element. This degradation destroys the sensing aptamer inhibiting the quantitative measurement of the target analyte and thus limits the application of E-AB sensors constructed with RNA aptamer. To circumvent this, we demonstrate that a biocompatible hydrogel membrane protects the RNA aptamer sensor surface from enzymatic degradation for at least 3 hours - a remarkable improvement over the rapid ( minutes) degradation of unprotected sensors. To demonstrate this, we characterize the response of sensors fabricated with representative DNA and RNA aptamers directed against the aminoglycoside antibiotic, tobramycin in blood serum both protected and unprotected by a polyacrylamide membrane. Furthermore, we find encapsulation of the sensor surface with the hydrogel does not significantly impede the detection ability of aptamer-based sensors. This hydrogel-aptamer interface will thus likely prove useful for the long-term monitoring of therapeutics in complex biological media.
\end{abstract}

Keywords: biosensor, electrochemical detection, biocompatible, aptamers, small molecule detection, serum

\section{Introduction}

Biosensors that can rapidly provide quantitative information about biomarker concentrations directly in complex sample media have the ability to revolutionize modern healthcare. This real-time molecular information in the healthcare setting could greatly enhance the caregiver's ability to efficiently administer treatment and maximize patient outcome. For example, the ability to rapidly diagnose biomarker levels for time-sensitive critical care issues such as infectious diseases [1] or sepsis [2] could provide accurate and actionable information to a clinician for immediate course of action and treatment [1-5]. In addition, the real-time monitoring of the pharmacokinetics of narrow therapeutic window of chemotherapeutics could also provide individualized treatment with improved patient outcome. The current cornerstones in "real-time" therapeutic monitoring involves the monitoring of patient symptoms during treatment [5]. Unfortunately, this information, which can take days to report, is transferred verbally and is open to interpretation by the patient and the clinician.

Electrochemical, aptamer-based (E-AB) sensors represent a promising platform to achieve real-time detection of relevant biomarkers as a result of their specificity, selectivity and sensitivity [6,7]. These sensors comprise singlestranded DNA or RNA aptamers that are attached to a sensing electrode surface and modified at the distal end with a redox active marker [8-11]. The aptamers are selected to bind a specific target [12], upon the addition of specific target, aptamer undergoes a conformation change [11], which changes the accessibility of the redox marker to the sensing electrode surface. Because the efficiency of electron transfer is dependent on the distance and collisional frequency of the redox marker, this conformation change is readily measured electrochemically[6,8-10,13-16]. As a result, E-AB sensors are specific (aptamer binding is specific) and selective (there are relatively few electroactive interferents), and thus DNA-based sensors can achieve detection directly in untreated serum $[6,15]$. RNA-based sensors however require 
significant serum pretreatment to achieve this detection $[8,16]$. Specifically, to date all E-AB sensors employing RNA aptamers require that serum either be filtered prior to use to remove any harmful nucleases or treated with an RNase inhibitor $[8,15,16]$. This type of sample pretreatment is not amenable for providing rapid, real-time diagnostic and treatment information.

While, most E-AB sensors described to date are DNA-based as a result of the inherent better stability of this biomolecule, some RNA aptamers have been demonstrated to exhibit better specificity and sensitivity. For example, the theophylline aptamer binds theophylline but not caffeine which only differ by a single methyl group [15]. RNA aptamers can even exhibit enantioselectivity[17]. Furthermore, there are only $\sim 20$ DNA aptamers reported in comparison to $\sim 90$ RNA aptamers [18]. Presumably, the ability of RNA to adopt more complex tertiary structure leads to better aptamer binding abilities and total number of aptamers reported. The lack of employment of RNA aptamers for detection in complex media, such as serum thus motivates the work within. Here we demonstrate that a biocompatible hydrogel membrane formed on the E-AB sensor surface can block access of harmful nucleases to the sensor surface and thus allow for RNA sensor function for at least 3 hours while still allowing for specific detection of analyte target. Our findings suggest that this strategy has the potential way to generate a general sensor platform, which mitigates sensor degradation to enable long-term, real-time detection directly in biological environments.

\section{Materials and Methods}

\subsection{Materials}

6-mercapto-1-hexanol (97\%) and tobramycin (Sigma Aldrich) were used as received. Sodium chloride, Trizma base, and magnesium chloride (Sigma Aldrich), were used to make a Tris buffer solution at concentrations of $100 \mathrm{mM}, 20 \mathrm{mM}$, and $5 \mathrm{mM}$ respectively. These salts were diluted with ultrapure water (Mili-Q Ultrapure Water Purification, Milipore, Billerica, MA) and used in electrochemical measurements unless otherwise noted. The 5'-thiol, 3'-methylene blue modified DNA and RNA aptamers (HPLC-purified, Biosearch Technologies, Inc. Novato, CA) were used as received. The DNA aptamer sequence used was 5' $\mathrm{HS}\left(\mathrm{CH}_{2}\right)_{6}$-GGG ACT TGG TTT AGG TAA TGA GTC CC-Methylene blue 3'[8]. The RNA aptamer sequence used was 5' $\mathrm{HS}\left(\mathrm{CH}_{2}\right)_{6}$-GGG ACU UGG UUU AGG UAA UGA GUC CC-Methylene blue 3'[19]. The aptamer probes were stored in 0.01M EDTA aqueous solution, pH 8.0 (Sigma Aldrich), when not in use. Tris-2-carboxyethyl-phosphine (TCEP) (Sigma Aldrich) was used to reduce the disulfide bond of the aptamers 5' end prior to immobilization on the gold electrode surface. Acrylamide $(40 \% \mathrm{w} / \mathrm{v})(\mathrm{BioRad})$, bis-acrylamide $(2 \% \mathrm{w} / \mathrm{v})$ (BioRad), TEMED (N,N,N,N-tetramethylethylenediamine) (Sigma Aldrich) and ammonium persulfate (Sigma Aldrich) were used in the synthesis of the polyacrylamide hydrogel. Fetal bovine serum (Thermo Scientific) served as a serum proxy.

\subsection{Electrochemical Aptamer Sensor Fabrication}

E-AB sensors were fabricated on $2 \mathrm{~mm}$ diameter polycrystalline gold electrodes (CH Instruments, Austin, TX). Electrode modification was performed using a previously described method [8]. In short, the electrodes were mechanically polished in a circular fashion on microcloth (Buehler) with a $1 \mu \mathrm{L}$ diamond suspension (Buehler) followed by an alumina oxide slurry (Buehler). The electrodes were rinsed and sonicated for $5 \mathrm{~min}$ between mechanical polishing steps. Following the mechanical polishing, electrodes were cleaned electrochemically through a variety of voltammetric scans in dilute sodium hydroxide and sulfuric acid solutions. This electrochemical cleaning consisted of cycling 500 times between $-0.4 \mathrm{~V}$ and $-1.35 \mathrm{~V}$ vs. $\mathrm{Ag} / \mathrm{AgCl}$ in $0.5 \mathrm{M} \mathrm{NaOH}$, cycling 10 times at scan rates varying from $4 \mathrm{~V} / \mathrm{s}$ to 0.1 $\mathrm{V} / \mathrm{s}$ at potentials from $-0.35 \mathrm{~V}$ to $1.5 \mathrm{~V}$ vs. $\mathrm{Ag} / \mathrm{AgCl}$ in $0.5 \mathrm{M} \mathrm{H}_{2} \mathrm{SO}_{4}$, by cycling 10 times from $0.2 \mathrm{~V}$ to $1.5 \mathrm{~V}$ vs. $\mathrm{Ag} / \mathrm{AgCl}$ in $0.1 \mathrm{M} \mathrm{H}_{2} \mathrm{SO}_{4}$ and $0.01 \mathrm{M} \mathrm{KCl}$, and cycling 10 times from $-0.35 \mathrm{~V}$ to $1.5 \mathrm{~V}$ vs. $\mathrm{Ag} / \mathrm{AgCl}$ in $0.05 \mathrm{M} \mathrm{H}_{2} \mathrm{SO}_{4}$. After cleaning, each electrode was incubated in a solution containing $200 \mathrm{nM}$ DNA in tris buffer for $1 \mathrm{~h}$. These electrodes were then rinsed with water and incubated in $3 \mathrm{mM}$ 6-mercapto-1-hexanol for $1 \mathrm{~h}$, which was diluted with buffer [8]. Rinsed electrodes were stored in a solution of Tris buffer prior to use.

\subsection{Electrochemical Measurements}

All electrochemical measurements were performed using a $\mathrm{CH}$ Instruments 620D Electrochemical Work Station $(\mathrm{CH}$ Instruments, Austin, TX). All measurements were performed in a three-electrode cell utilizing an $\mathrm{Ag} / \mathrm{AgCl}$ reference electrode and a platinum wire counter electrode. Square wave voltammetry parameters were as follows: a pulse 
amplitude of $25 \mathrm{mV}$, frequency of $60 \mathrm{~Hz}$ and step width of $1 \mathrm{mV}$ or $4 \mathrm{mV}$. All measurements were performed in a glass cell with $3 \mathrm{~mL}$ of Tris buffer or $3 \mathrm{~mL}$ of fetal bovine serum (Sigma Aldrich).

\subsection{Hydrogel Membrane Polymerization and Utilization}

Encapsulation of the sensor surface with a protecting hydrogel was performed after sensor calibration with a tobramycin titration. This titration ensures that the sensors quantitatively respond to the presence of target analyte (tobramycin). A polyacrylamide solution was prepared to contain $2 \mathrm{~mL}$ of acrylamide solution $(35.3 \%), 0.2 \mathrm{~mL}$ of the bis-acrylamide solution $(0.2 \%), 15 \mu \mathrm{L}$ of $(0.7 \%)$ TEMED, and $50 \mu \mathrm{L}$ of a $5 \mathrm{mM}$ ammonium persulfate solution. The solution was inverted gently to mix the reactants. The freshly prepared pre-polymerized solution was then dispensed in $\sim 10 \mu \mathrm{L}$ aliquots onto each electrode surface. Sensors were left in a humidity chamber until the solution polymerized ( $\sim 60 \mathrm{~min})$.

\section{Results and Discussion}

\subsection{Performance of Bare Sensors in Buffer}

As a representative test-bed sensor platform, we fabricated E-AB sensors for the sensitive detection of the aminoglycoside antibiotic tobramycin [8]. We chose this target because both RNA and DNA aptamers exist as demonstrated through the development E-AB sensors for the detection of this target recently published by Rowe et al.[8]. In this same report, several strategies were employed to protect RNA from degradation by nucleases including methylation of the 2'-hydroxyl groups and conversion of the sequence to a DNA strand. While the DNA version maintained tobramycin-sensing ability, it did so with a lower affinity. Ultimately, the investigators had to resort to ultracentrifugation of the serum to allow RNA sensor use.

RNA and DNA aptamer-based sensors utilizing anti-aminoglycoside aptamer rapidly and reversibly respond to the presence of the aminoglycoside antibiotic, tobramycin (Fig. 1). As shown in Figure 1, the presence of tobramycin causes a decrease in measured squarewave peak current for RNA and DNA-based sensors similar to previously reported [8]. With a simple 5 seconds water rinse the signal is regenerated to within at least $95 \%$ of its original value.
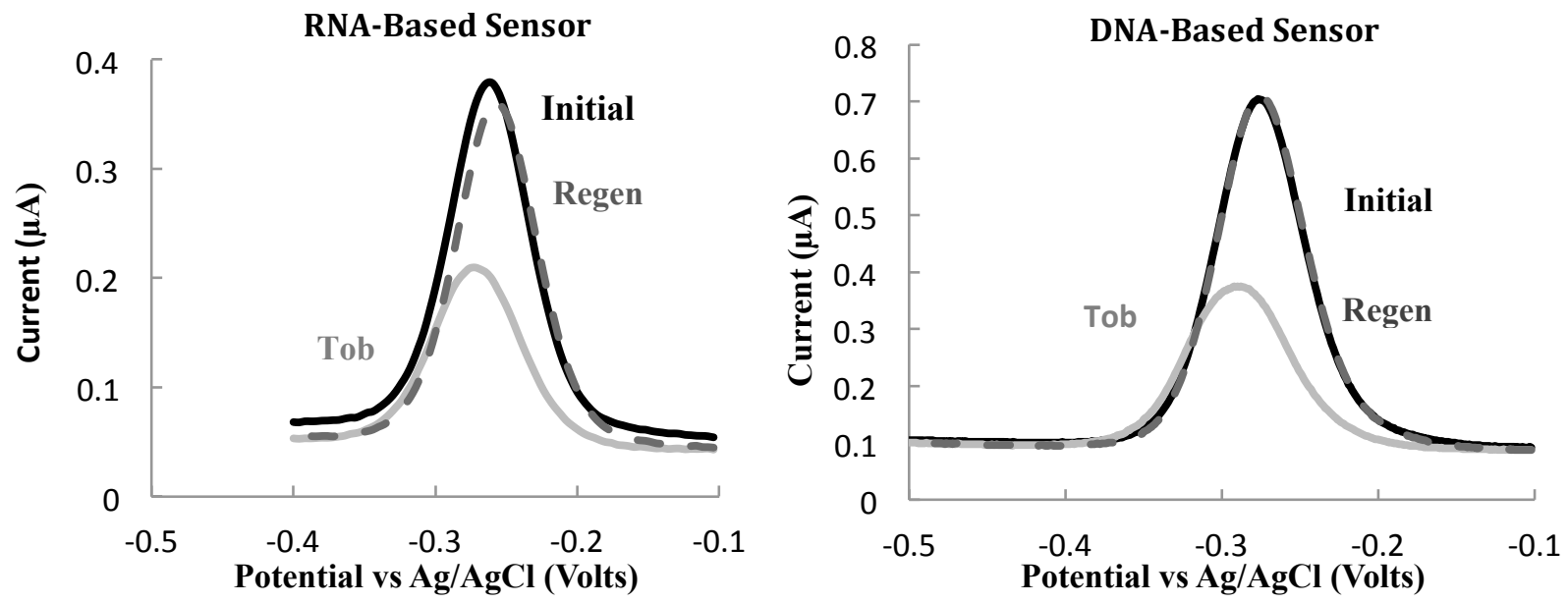

Figure 1: E-AB aminoglycoside sensors are rapid and reusable. RNA- (left) and DNA-based (right) sensors exhibit a decrease in peak current upon the addition of saturating levels of tobramycin (Tob - $2 \mathrm{mM}$ ). However in simply rinsing the electrode surface with water the initial signal can be regenerated (regen). This response also occurs rapidly, and is equilibrated within the several seconds it takes between each voltammetric scan.

The sensors also respond quantitatively to the presence of tobramycin (Fig. 2). The percent decrease in signal (signal suppression) provides indication of the amount of tobramycin present in solution. These data can be fit to a Langmuir adsorption isotherm describing non-competing binding to a finite number of binding sites $[9,20]$. Unlike previously reported, the DNA aptamer sensor out performs the RNA sensor displaying a $45 \%$ signal change at saturating target concentration $(2 \mathrm{mM})$ with a $160 \pm 22 \mu \mathrm{M}$ dissociation constant compared to the RNA sensor which has a $35 \%$ maximum signal change with a $306 \pm 70 \mu \mathrm{M}$ dissociation constant (Fig. 2) [8]. The discrepancy in which the DNA 
sequence displays better affinity for tobramycin could be a result of unoptimized surface conditions. That is, observed binding affinity is sensitive to the packing density of the probe aptamers on the electrode surface [9], and sensors employed here were not optimized in terms of this parameter.

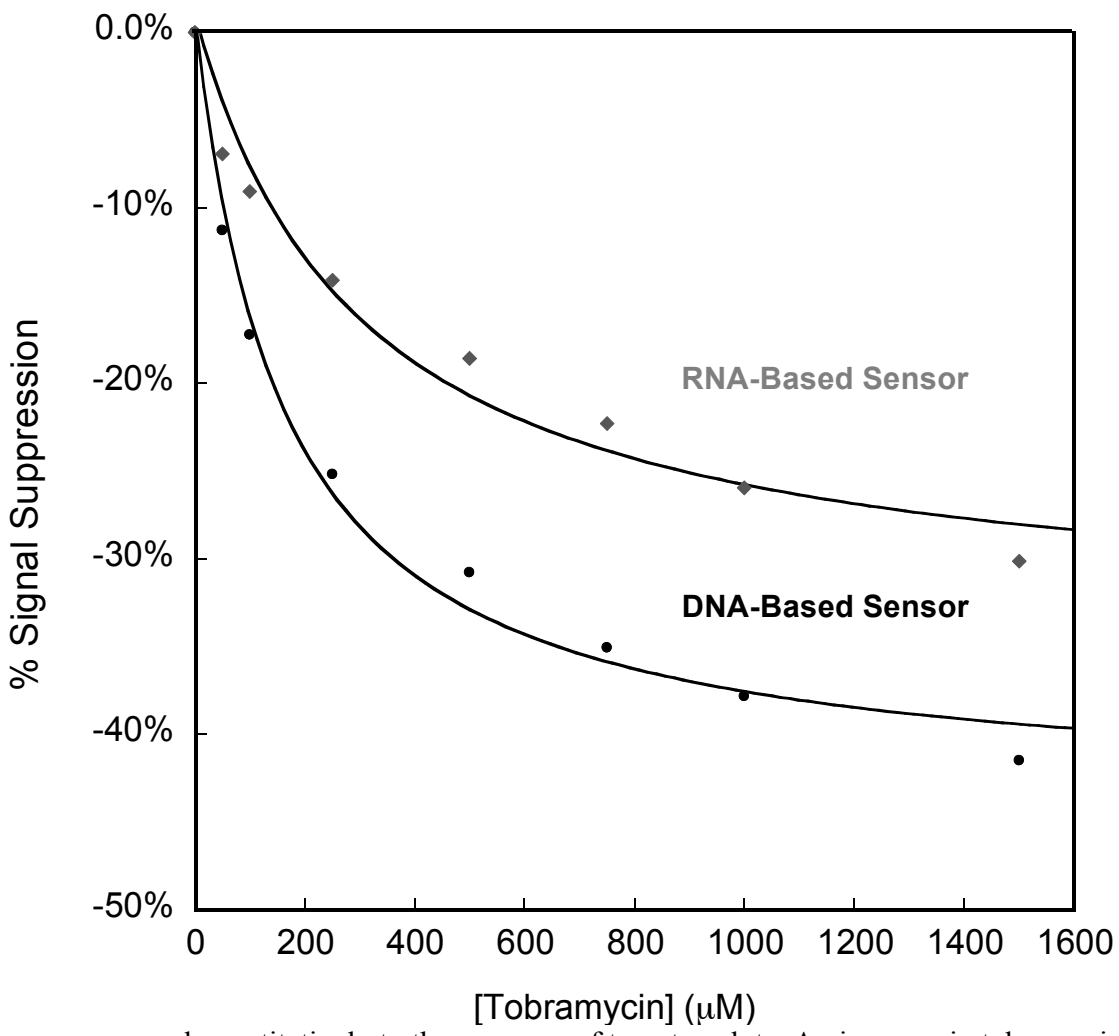

Figure 2: E-AB sensors respond quantitatively to the presence of target analyte. An increase in tobramycin cooncentration causes larger decrease in peak current (expressed as percent signal suppression) for both the DNA and RNA aptamer-based sensors. They exhibit a $160 \pm 22 \mu \mathrm{M}$ and $306 \pm 70 \mu \mathrm{M}$ observed binding affinity respectively when fit to a Langmuir isotherm. Signal suppression is displayed as the average percent signal suppression for three DNA-based and three RNA-based sensors.

\subsection{Performance of Bare Sensors in Serum}

Anti-aminoglycoside E-AB sensors utilizing the DNA aptamer are selective, capable of tobramycin detection directly in undiluted serum. Specifically, when challenged with saturating target concentration in serum, E-AB sensors with DNA aptamers exhibit $\sim 60 \%$ signal suppression (data not shown). Conversely, RNA-based sensors do not perform when challenged in undiluted serum, presumably a result of degradation of the RNA aptamer. To further characterize the origin of this failure mode, we subjected each sensor (RNA and DNA) to repeated scans in a serum sample while monitoring peak current as a function of time (Fig. 3). E-AB sensors fabricated with RNA aptamer sensing elements exhibit a rapid decrease in peak current, leveling off at $\sim 70 \%$ signal loss after $60 \mathrm{~min}$. Alternatively, DNA-based sensors remain, within error, unchanged over the same 200 minutes. The measured peak current is a result of the reversible twoelectron, one-proton reduction of methylene blue. Any decreases in this peak current could result from either physical steric blocking of access to the electrode surface (e.g., the nonspecific adsorption of serum proteins) or loss of the redox molecule (e.g., desorption of thiol or degradation of the sensing element). Because DNA-based sensors show no decrease in measured peak current, we rule out non-specific adsorption as the cause of sensor failure. Alternatively, we hypothesize through this data and literature reports that RNA degradation by RNase activity is the major cause of sensor failure $[16,21]$. 


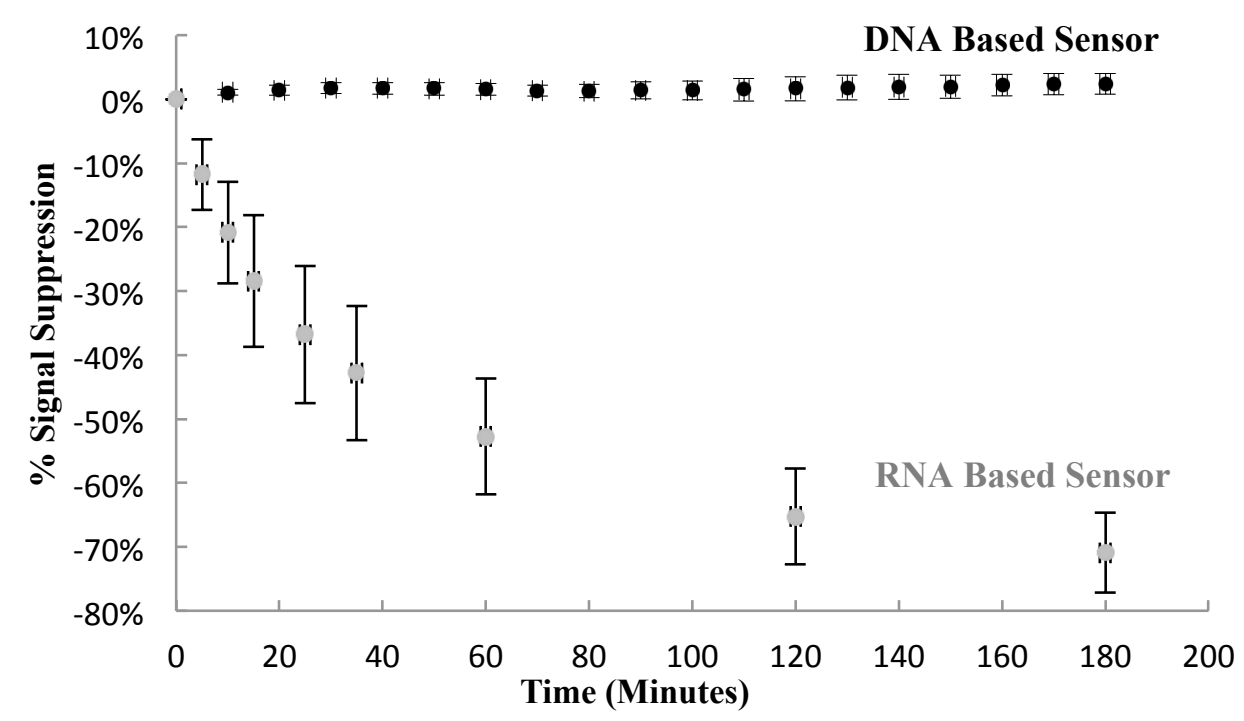

Figure 3: When employed in undiluted blood serum, RNA aptamer sensors exhibit rapid degradation in comparison to DNA aptamerbased sensors. Monitoring peak current as a function of time deployed in serum provides a qualitative picture of both the accessibility of the electrode surface to redox marker (which can change as a result of nonspecific adsorption of serum proteins) and the total amount of redox marker tethered to the surface (which can change if the aptamer degrades thus liberating the redox probe). Over the same 200-minute experiment, the DNA-based sensor remains virtually unchanged, while the RNA-based sensor rapidly loses $\sim 70 \%$ of its signal. Because the DNA surface shows no susceptibility to nonspecific adsorption of protein, the loss in signal for the RNA surface is presumed to be a result of enzymatic/chemical degradation of the RNA sensing element.

\subsection{Performance of Hydrogel Coated Sensors in Buffer and Serum}

Previous research reports demonstrate that RNA-based E-AB sensors will function in serum after significant pretreatment to eliminate the presence of nucleases. Specifically, Rowe et al. and Feraptonova et al. report filtering the serum via ultracentrifugation with $\sim 3000 \mathrm{kDa}$ molecular weight cutoff filters to remove serum proteins $[8,15,16]$. Alternatively Feraptonova demonstrate that treating serum biochemically to inhibit nuclease dramatically imrpoves RNA-based E-AB sensor performance in serum.

Our approach is to encapsulate the RNA-based sensor with a biocompatible membrane to prevent nucleases from reaching the sensor surface thus protecting the surface attached RNA. With this in mind, after sensor function was validated, a polyacrylamide membrane was deposited onto the modified-electrode surface. Upon polymerization the hydrogel membrane can be characterized by its mesh (or pore) size. This physical characteristic of the hydrogel is controlled via the concentration of the monomer unit (acrylamide) and crosslinker (bis-acrylamide) employed during hydrogel polymerization. Given our polymerization protocol ( $35.3 \%$ acrylamide and $0.2 \%$ bis-acrylamide), the mesh size was estimated using Flory-Rehner theory and equilibrium swelling measurements to be $\sim 40 \mathrm{~nm}[22,23]$.

E-AB sensors encapsulated with a protecting hydrogel membrane supports sufficient E-AB sensor function. After the hydrogel membranes were allowed to polymerize, the encapsulated sensors were tested in buffer for their ability to bind tobramycin (Fig. 4). When challenged with tobramycin, the sensor yields a $61 \%$ and $60 \%$ signal change for the DNA and RNA-based sensors respectively (compared to the $45 \%$ and $35 \%$ observed from bare electrodes). Of note, upon submersing the sensor into Tris buffer, the peak current increased until reaching equilibrium after $\sim 20 \mathrm{~min}$. Similarly, after the addition of $2 \mathrm{mM}$ tobramycin, the sensor signaling equilibrated after $\sim 20 \mathrm{~min}$. This equilibration time is a result of the solution inside the hydrogel equilibrating with the external solution. While this is undesirable for rapid, real-time detection, the membranes used within are not optimized in terms of both membrane thickness and mesh size. We anticipate that doing so will improve the time response of the sensors and as such this is currently under investigation. Finally, after a $\sim 90$ min submersion in buffer without target, the sensors regenerated to $\sim 60-70 \%$ of their original signal (Fig. 4). The origin of the less than optimal regeneration again is presumed to be a function of the physical properties, of which we are actively investigating. 

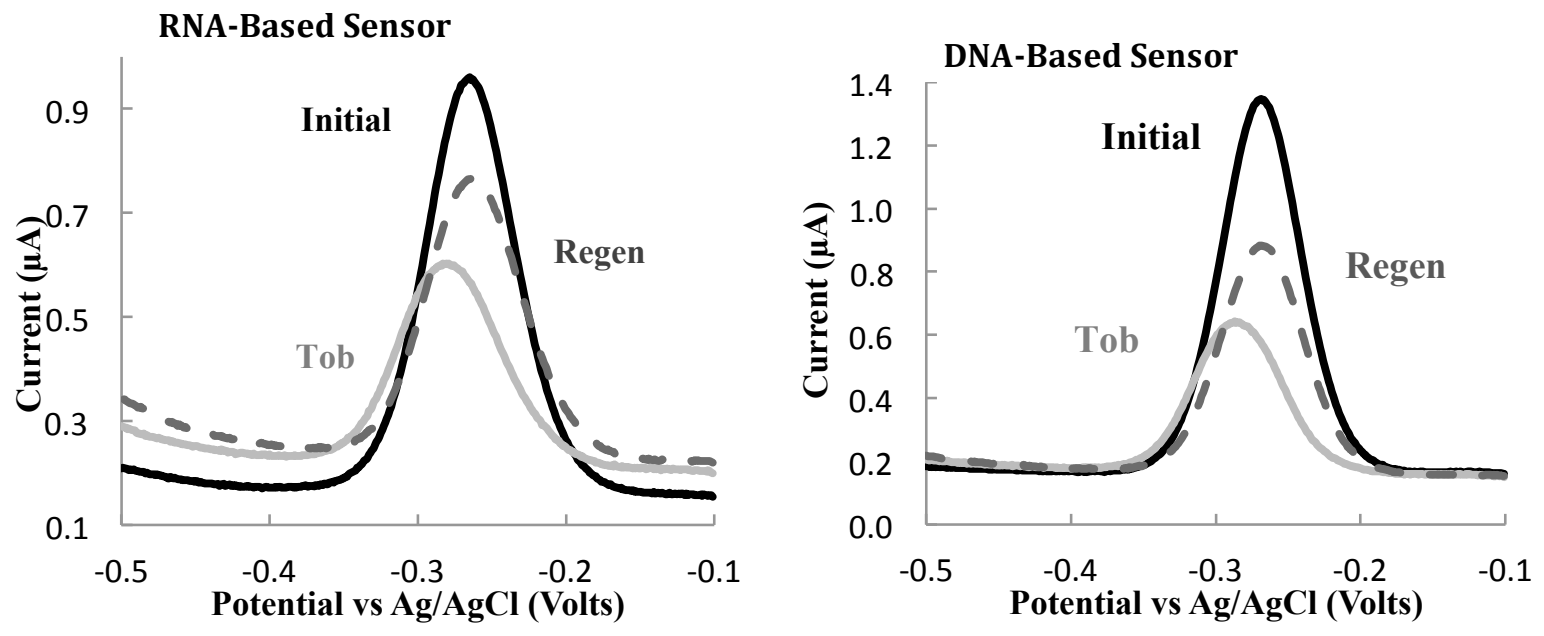

Figure 4: Hydrogel-protected RNA (left) and DNA (right) aptamer-based sensors respond effectively to the presence of specific target. Both sensors exhibit a decrease in peak current upon the addition of $2 \mathrm{mM}$ tobramycin with a polyacrylamide membrane on the sensor surface after equilibrating for $\sim 20 \mathrm{~min}$. In rinsing the electrode surface with water the initial signal can be regenerated to within $\sim 60-70 \%$.

To test the protection abilities of the polyacrylamide membrane the RNA-based E-AB sensors were immersed directly in undiluted serum with and without a polyacrylamide membrane (Fig. 5). As described above, square wave voltammetric peak current was monitored as a function of time to qualitatively test the integrity of the sensing monolayer. As such, the sensors were placed in fetal bovine serum and were tested every 10 minutes for 3 consecutive hours. The percent change was calculated with respect to the initial square wave voltammogram in serum (Fig. 5). After $\sim 50$ minute equilibration period (where buffer is exchanged for serum in the membrane), E-AB sensors with RNA show almost no degradation for $3 \mathrm{~h}$, in comparison to an uncoated sensor, which decays by $70 \%$ within the first hour (Fig. 3 and Fig. 5).

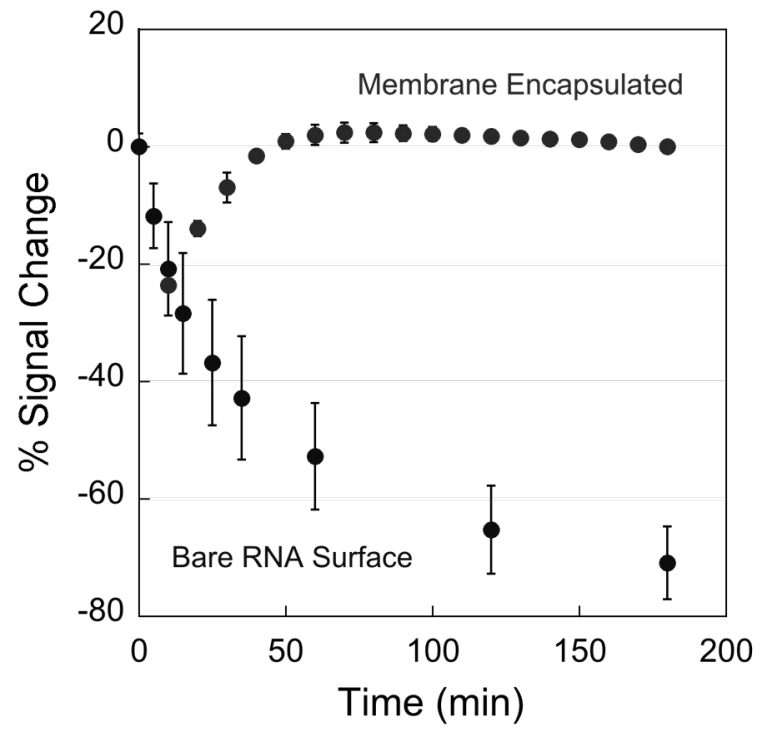

Figure 5: Incorporation of a protecting hydrogel layer dramatically improves the stability of an RNA-based E-AB sensor in serum. This graph shows the peak current percent signal change with respect to the initial peak current measured in fetal bovine serum over time. This displays RNA sensor signals' stability (with membrane) and degradation (without membrane) for 200 minutes. 


\section{Conclusions}

Electrochemical aptamer-based sensors that employ RNA sensing elements suffer from sensor failure upon employment in a complex sample matrix such as blood serum. The failure mode appears to be a product of enzymatic degradation of the actual sensing element (RNA) as opposed to nonspecific adsorption of serum proteins onto the sensor surface. This is supported by the observation that E-AB sensors fabricated with DNA aptamers are relatively robust on long time scales ( hours) employed in undiluted serum. Several literature reports attempt to overcome this limitation by significant sample pretreatment either through ultracentrifugation [8], biochemical treatment to inhibit nuclease activity [8,16], or RNA 2' hydroxyl modification via attempting to protect this hydroxyl or replacing it with a fluorine [8,24].

Here, we demonstrate a suitable alternative method to allow RNA-based sensor function directly in serum via the encapsulation of the sensor surface with a protecting, biocompatible polyacrylamide hydrogel membrane. The unoptimized hydrogel membrane dramatically improves the stability of the RNA-based sensors when employed in serum. In addition to protecting the RNA sensing element for at least $\sim 3$ hours, the new hybrid membrane/sensor interface still supports efficient sensor signaling. The encapsulated sensors still recognize and give response to the addition of the tobramycin target, albeit with a diminished temporal response. Optimization of the physical parameters of the membrane, which allow rapid analyte to diffusion, while still inhibiting the enzymatic attack of the RNA aptamers, is still needed and is under investigation. Nonetheless, these preliminary findings suggest that our method of hydrogel encapsulation of sensor surface will enable to use of RNA based E-AB sensors directly in complex media rendering them suitable for rapid diagnostics.

\section{Acknowledgements}

This work was supported by UMBC Startup.

\section{References}

[1] Shu, C.-C., Wu, M.-F., Hsu, C.-L., Huang, C.-T., Wang, J.-Y., Hsieh, S.-L., Yu, C.-J., Lee, L.-N., and Yang, P.-C., “Apoptosisassociated biomarkers in tuberculosis: promising for diagnosis and prognosis prediction.," BMC infectious diseases 13,45 (2013).

[2] Santini, J., Milano, G., Thyss, A., Renee, N., Viens, P., Ayela, P., Schneider, M., and Demard, F., "5-FU therapeutic monitoring with dose adjustment leads to an improved therapeutic index in head and neck cancer.," British journal of cancer 59(2), 287-90 (1989).

[3] Kibe, S., Adams, K., and Barlow, G., "Diagnostic and prognostic biomarkers of sepsis in critical care.," The Journal of antimicrobial chemotherapy 66 Suppl 2, ii33-40 (2011).

[4] Pascual, A., Calandra, T., Bolay, S., Buclin, T., Bille, J., and Marchetti, O., "Voriconazole therapeutic drug monitoring in patients with invasive mycoses improves efficacy and safety outcomes.," Clinical infectious diseases : an official publication of the Infectious Diseases Society of America 46(2), 201-11 (2008).

[5] Basch, E., Artz, D., Dulko, D., Scher, K., Sabbatini, P., Hensley, M., Mitra, N., Speakman, J., McCabe, M., et al., "Patient online self-reporting of toxicity symptoms during chemotherapy.," Journal of clinical oncology : official journal of the American Society of Clinical Oncology 23(15), 3552-61 (2005).

[6] Swensen, J. S; Xiao, Y; Ferguson, B. S; Lubin, A. A; Lai, R. Y; Heeger, A. J; Plaxco, K. W; Sho, H.T., “Continuous, Real-Time Monitoring of Cocaine in Undiluted Blood Serum via a Microfluidic, Electrochemical Aptamer-Based Sensor," Journal of American Chemical Society 131(1), 4262-4266 (2010).

[7] Zuo, X., Song, S., Zhang, J., Pan, D., Wang, L., and Fan, C., "A target-responsive electrochemical aptamer switch (TREAS) for reagentless detection of nanomolar ATP.," Journal of the American Chemical Society 129(5), 1042-3 (2007). 
[8] Rowe, Aaron, A., Miller, Erin, A., Plaxco, Kevin, W., "Reagentless Measurement of Aminoglycoside Antibiotics in Blood Sereum via an Electrochemical, Ribonucleic Acid Aptamer-Based Biosensor," Analytical chemistry 82(17), 7090-7095 (2011).

[9] White, R.J., and Plaxco, K.W., "Engineering New Aptamer Geometries for Electrochemical Aptamer-Based Sensors," Proc. SPIE 7321, 732105-732117 (2009).

[10] Xiao, Y., Lubin, A. A., Heeger, A.J., and Plaxco, K.W., "Label-free electronic detection of thrombin in blood serum by using an aptamer-based sensor.," Angewandte Chemie (International ed. in English) 44(34), 5456-9 (2005).

[11] White, R. J., Phares, N., Lubin, A. A., Xiao, Y., Plaxco, K.W., “Optimization of Electrochemical Aptamer-Based Sensors via Optimization of Probe Packing Density and Surface Chemistry," Langmuir 24(18), 10513-10518 (2009).

[12] Iliuk, A.B., Hu, L., and Tao, W.A., “Aptamer in bioanalytical applications.,” Analytical Chemistry 83(12), 4440-52 (2011).

[13] Xiao, Y., Uzawa, T., White, R. J., DeMartini, D., Plaxco, K.W., “On the Signaling of Electrochemical Aptamer-Based Sensors: Collision- and Folding-Based Mechanisms," Electroanalysis 21(11), 1267-1271 (2010).

[14] Jiang, L., and Patel, D.J., "Solution structure of the tobramycin-RNA aptamer complex.," Nature Structural Biology 5(9), 769-74 (1998).

[15] Ferapontova, E.E., Olsen, E.M., and Gothelf, K. V, "An RNA aptamer-based electrochemical biosensor for detection of theophylline in serum.," Journal of the American Chemical Society 130(13), 4256-8 (2008).

[16] Ferapontova, E.E., and Gothelf, K. V., “Optimization of the Electrochemical RNA-Aptamer Based Biosensor for Theophylline by Using a Methylene Blue Redox Label," Electroanalysis 21(11), 1261-1266 (2009).

[17] Geiger, T., Cox, J., and Mann, M., "Proteomic Changes Resulting from Gene Copy Number Variations in Cancer Cells," PLOS Genetics 6(9), (2010).

[18] Carrasquilla, C., Lau, P.S., Li, Y., and Brennan, J.D., "Stabilizing Structure-Switching Signaling RNA Aptamers by Entrapment in Sol-Gel Derived Materials for Solid-Phase Assays," Journal of the American Chemical Society 134(26), 10998-11005 (2012).

[19] Wang, Y; Killian, J; Hamasaki, K; Rando, R.R., "RNA Molecules That Specifically and Stoichiometrically Bind Aminoflycoside Antibiotics with High Affinities," Biochemistry 35(38), 12338-12346 (1996).

[20] White, R. J., Phares, N., Lubin, A. A., Xiao., Plaxco, K. W., “Optimization of Electrochemical Aptamer-Based Sensors via Optimization of Probe Packing Density and Surface Chemistry" 24(18), 10513-10518 (2008).

[21] Ferapontova, E.E., Olsen, E.M., and Gothelf, K. V, “An RNA aptamer-based electrochemical biosensor for detection of theophylline in serum.," Journal of the American Chemical Society 130(13), 4256-8 (2008).

[22] Baier Leach, J., Bivens, K. a, Patrick, C.W., and Schmidt, C.E., "Photocrosslinked hyaluronic acid hydrogels: natural, biodegradable tissue engineering scaffolds.," Biotechnology and Bioengineering 82(5), 578-89 (2003).

[23] Wu, C., Quesada, M. a, Schneider, D.K., Farinato, R., Studier, F.W., and Chu, B., "Polyacrylamide solutions for DNA sequencing by capillary electrophoresis: mesh sizes, separation and dispersion.," Electrophoresis 17(6), 1103-9 (1996).

[24] Ulrich, H., Martins, A. H. B., Pesquero, J. B. "RNA and DNA Aptamers in Cytomics Analysis," Cytometry Part A: the Journal of the International Society for Analytical Cytology 59(2), 220-231 (2004). 